\title{
The Bogolyubov method of quasiaverages solves the problem of pressure fluctuations in the Gibbs statistical mechanics
}

\author{
Yu.G. Rudoy* \\ People's Friendship University, 117198, 6 Miklukho-Maklaya Str., Moscow, Russia
}

Received July 23, 2009, in final form September 3, 2009

\begin{abstract}
The long-standing and highly non-trivial problem of calculating the pressure fluctuations in the Gibbs equilibrium statistical mechanics is fully revised. The previous attempts are critically analyzed and it is shown that the application of the ideas by Bogolyubov gives the full and unambiguous solution of this problem. The crucial role is played by the Bogolyubov's idea of quasiaverages (or rather quasidynamic) quantities - specifically, the pressure $P$ and dynamic compressibility $\Psi$. The virtual conjugate field which eliminates the translational invariance of the Hamilton function $H$ in the limit $\varepsilon \rightarrow 0$ is the singular potential of the impenetrable walls of the container. The general relations for $P$ and $\Psi$ in terms of the derivatives of $H$ are obtained and some examples are studied - i. e., the cases of the ideal vs. non-ideal as well as of uniform vs. non- and quasi-uniform (in Euler sense) Hamilton function $H$ describing the given system.
\end{abstract}

Key words: Gibbs equilibrium statistical mechanics, Bogolyubov's quasiaverages, pressure fluctuations, relativistic ideal gas

PACS: $05.70 .-a, 05.30 .-d, 05.40 .-a$

\section{Introduction}

The problem of the equilibrium pressure fluctuations is one of the oldest and most difficult problems in classical statistical mechanics. In 1902 Gibbs [1], Ch.VII, proved his famous Lemma and wrote down the appropriate expression, which includes the quantity named by him the "dynamical compressibility"; see also Fowler [2], Hill [3], Kittel [4], Terletzky [5] (more precise definitions are given below).

Many attempts have been made to calculate this quantity - e. g., by Fowler [2], Wergeland [6], Münster [7,8], M. Klein [9], but no fully satisfactory results have been obtained even for the simplest case of the "ordinary" - i. e., non-relativistic ideal gas (usually called Maxwell gas). Some "pessimistic" point of view was expressed in [3], Ch. $4, \S 19$ (see also [5], §69), where the calculation of the pressure fluctuations was connected with the detailed knowledge of the kind of forces acting between the gas particles and the container walls. Unfortunately, some attempts $[2,3]$ to follow this route brought to physically unsatisfactory - i. e., divergent - results. Here it is appropriate to recall the argument by Maxwell that the gas in the container even relaxes to the state of thermal equilibrium quite independent of the physical properties of the walls; clearly, it should be the more so with the fluctuations when this state is already achieved.

Moreover, it was even claimed (see, e. g., [4], Ch. 11) that the solution of the problem of the Gibbs pressure fluctuations is generally outside the scope of the equilibrium theory, so all these failures were sometimes considered as the inconsistency of the Gibbs approach as a whole. Indeed, in the framework of any complete theory of probabilistic nature it should be possible to calculate not only the average values, but also the correlations and dispersions of any dynamical variables so why should the pressure be an exclusion?

The situation becomes even more involved by noting that some of the physically acceptable results for the pressure fluctuations obtained in [7-9] refer in fact not to the Gibbs approach

\footnotetext{
*E-mail: rudikar@mail.ru
} 
itself, but to the Einstein one, which is referred to as "quasi-thermodynamic" by Landau and Lifshitz [10]. These approaches significantly differ in the choice of thermodynamic variables fixed while calculating; e. g., in the case of pressure fluctuations this is the entropy in Einstein's approach, though this is the volume in Gibbs's ensemble approach (more details are given in [15]).

The way out was actually outlined in 1946 by Bogolyubov [11], who used the coordinate scale transformation in order to connect the thermodynamic pressure with the dynamical quantities - namely, with the first derivatives of the Hamilton function and the particles' pair distribution function. Later on in 1971 Zubarev [12] obtained an analogous expression for the dynamical (i. e., still thermally unaveraged) pressure defined only on the phase space variables.

In fact, in [12] there was implicitly used an idea of quasiaverages - or, in this case it is better to say, quasidynamic quantities - which was also formulated by Bogolyubov [13] in 1961. From the computational point of view Zubarev's result became possible by virtue of a direct use of the generalized function (in this case - the singular potential of container walls) following the technique outlined by Vladimirov [14].

But it was only in 2000 when the present author with Sukhanov [15] succeeded to extend the Bogolyubov-Zubarev approach and for the first time obtain the general expression for the Gibbs "dynamical compressibility" in terms of the second derivatives of the Hamilton function. This expression is quite universal and is valid for any reasonable kind of kinetic energy and interaction potential, but only the Maxwell gas was considered in [15] as an example.

Later on in 2007 the present author with Keita [16] and also with Keita and Rybakov [17] extended these results to the ultra-relativistic ideal gas (so-called Wien gas) and, more generally, to the ideal gas with any uniform (in Euler sense) dependence of the Hamilton function upon the (quasi)particle momentum. Finally, the most general case of non-uniform Hamilton function - of Lorentz as well Lorentz-violated form [16] - was considered for the classical ideal gas.

In the present paper the pressure fluctuation problem in equilibrium classical statistical mechanics is fully revisited and the generalization of the Bogolyubov-Zubarev result is given. The presentation is founded on the fundamental Bogolyubov's idea of quasiaverages (in our case rather quasidynamic) physical observables along with the direct use of the strictly infinite potential of the walls confining the gas. It is just this potential which violates the uniform space symmetry of the initial Hamilton function and allows us to define the pressure and its fluctuations in the correct and unambiguous form.

Thus, using the seminal Bogolyubov's ideas, we managed to show the logical and computational completeness of the Gibbs statistical mechanics, which was occasionally brought to doubt especially in connection with the problem of pressure fluctuations.

\section{Formulation of the problem}

In short, the technical problem is the following one. The equilibrium pressure fluctuations $\left\langle(\Delta P(\Gamma))^{2}\right\rangle$ are defined in a standard way as $\left\langle(\Delta P(\Gamma))^{2}\right\rangle=\left\langle(P(\Gamma))^{2}\right\rangle-\langle P(\Gamma)\rangle^{2}$, where $\Gamma=\{q, p\}$ is the phase space with the coordinates $q$ and momenta $p ;\langle\ldots\rangle$ denotes the canonical averaging for the system in the isothermal-isochoric ensemble with fixed values ${ }^{1}$ of $\beta$ and $V$.

Following Gibbs [1], if the Hamilton function $H(\Gamma)$ for the dynamic system is given, then

$$
\begin{gathered}
\langle\ldots\rangle=Z^{-1}(\beta, V) \int \mathrm{d} \Gamma \exp [-\beta H(\Gamma)](\ldots), \\
Z(\beta, V)=\int \mathrm{d} \Gamma \exp [-\beta H(\Gamma)], \quad \Phi(\beta, V)=\ln Z(\beta, V),
\end{gathered}
$$

where the partition function $Z(\beta, V)$ is supposed to be finite and strictly positive, so that the Massieu-Planck thermodynamic potential $\Phi(\beta, V)$ does exist. The latter is usually a smooth function of $\beta$ and $V$, so there also exist the relevant thermodynamic derivatives, in particular equilibrium (i. e., isothermal) pressure $P(\beta, V)$ and compressibility $\chi(\beta, V)<0$ :

$$
P(\beta, V)=(1 / \beta)[\partial \Phi(\beta, V) / \partial V], \quad \chi(\beta, V) \equiv \partial P(\beta, V) / \partial V=(1 / \beta)\left[\partial^{2} \Phi(\beta, V) / \partial V^{2}\right] ;
$$

\footnotetext{
${ }^{1}$ The value of $\beta$ is introduced by the canonical distribution function whereas $V$ - by the restriction of the region of $\Gamma$.
} 
the expressions (2) are known in thermodynamics as thermic equations of state.

According to the famous Gibbs lemma [1] (Ch. VII, equations (252), (255), see also [3] and [5]), the equilibrium pressure fluctuations $\left\langle(\Delta P(\Gamma))^{2}\right\rangle$ are given by the expression

$\beta\left\langle(\Delta P(\Gamma))^{2}\right\rangle=\chi(\beta, V)+\Psi(\beta, V), \quad \chi(\beta, V)=\partial\langle P(\Gamma)\rangle / \partial V, \quad \Psi(\beta, V)=-\langle\partial P(\Gamma) / \partial V\rangle$,

or, following Gibbs and introducing the additional dynamic quantity $\Psi(\Gamma)$,

$$
\Psi(\beta, V)=\langle\Psi(\Gamma)\rangle, \quad \Psi(\Gamma)=-\partial P(\Gamma) / \partial V=\partial^{2} H(\Gamma) / \partial V^{2}, \quad P(\Gamma)=-\partial H(\Gamma) / \partial V
$$

Gibbs referred to the quantity $\Psi(\Gamma)$ as dynamic compressibility, but gave no example of its calculation; in general, the calculation of quantities in (3) and (4) consists of two stages: firstly, the adequate definition of $P(\Gamma)$ and $\Psi(\Gamma)$, and secondly - their correct averaging according to (1).

Note that for the pressure, the first stage may be in fact bypassed due to the first of equation (2) along with definitions in $(1)$, and thus the pressure $P(\beta, V)$ is called the "thermodynamic" average; on the contrary, though according to $(3) \Psi(\beta, V)$ also belongs to the set of Gibbs's averages, it is a "non-thermodynamic" one because it needs a direct calculation according to (1).

Further, in order to fulfill the conditions of thermodynamic stability relative to the external mechanical disturbance, it is necessary for $\left\langle(\Delta P(\Gamma))^{2}\right\rangle$ to be positive which requires $\Psi(\beta, V)$ not only to be positive but also to exceed $-\chi(\beta, V)$. Hence $\Psi(\beta, V)$ cannot be equal to $-\chi(\beta, V)$ which means (with account of $(3)$ and $(4))$ that the operation $\langle\ldots\rangle$ is in general not permutable with the operation $\partial / \partial V$ : just this circumstance is of decisive significance for further presentation.

It is worth noting that due to the Gibbs lemma the expressions analogous to (3) also hold for the equilibrium thermal fluctuations of other (thermo)dynamic quantities - e. g., energy $H$ or generalized force $A=-\partial H / \partial a$. In all cases the relevant derivatives in the Gibbs lemma refer to the (thermo)dynamically conjugate variables ${ }^{2}$, but in cases for $H$ and $A$ the terms $\partial H(\Gamma) / \partial \beta$ and $-\partial A / \partial a=\partial^{2} H / \partial a^{2}$ fully disappear and thus no difficulties arise at all. Indeed, the energy $H(\Gamma)$ is a pure dynamic variable and so - by definition - does not depend upon thermal parameter $\beta$, whereas variables $A$ and $a$ are mutually independent and enter $H(\Gamma)$ in the bilinear form $(-A a)$.

Quite different situation takes place for the pair of relevant (thermo)dynamically conjugate variables - the pressure $P$ and volume $V$. Strictly speaking, all the derivatives of the energy $H(\Gamma)$ upon volume $V$, entering the definitions (4), are identically zero by definition. Indeed, all the quantities $H(\Gamma), P(\Gamma)$ and $\Psi(\Gamma)$ in (4) are of pure dynamic nature and do not contain the kinematical parameter $V$. These quantities are defined throughout the whole phase space $\Gamma$ for the "free" system without any "walls" (therefore, for $V \rightarrow \infty$ ), while the finite value $V$ enters only on the final stage - i. e., after the averaging procedure when the proper account for confinements (if any) of kinematic nature in the system's phase space is already made in the main definition (1).

It is instructive to recall here, that the method of quasiaverages was created by Bogolyubov [11] just in order to cope with those frequently encountered problems, when the symmetry of the Hamiltonian $H$ of the physical system is higher than the symmetry of the ground state or of the state of thermal equilibrium of the given system. In these cases the formal calculations (1) prescribed by the Gibbs statistical mechanical approach [1] (as well as by its quantum generalization) give unphysical zero results for ordinary average values. It was convincingly shown by Bogolyubov [11], that the reason lies in the existence of some kind of degeneration in the system's energy, so the notion of quasiaverages appeared to be appropriate and unavoidable in order to obtain physically meaningful results.

Note that the term "degeneration" is fully deprived here of any "quantum" sense and is used only to designate the presence of some additional symmetry in $H$ (e. g., relative to the shifts or rotations in configuration part of the phase space $\Gamma$ ). The ingenious - though almost "obvious" idea by Bogolyubov was to remove this "degeneration" by means of relevant conjugate infinitesimal "external field" (in a broad sense) before the averaging procedure is carried out and then, after all calculations are made, fully eliminate this field ${ }^{3}$.

\footnotetext{
${ }^{2}$ For $H$ and $A$ these are the inverse temperature $\beta=1 / k_{\mathrm{B}} T$ and the relevant generalized parameter $a$.

${ }^{3}$ Spoken figuratively, the quasiaverages are very much like such fiction characters as Moor of Venice or the Cheshire cat.
} 
To be specific, in our case the Hamilton function $H(p, q) \equiv H(\Gamma)$ describing the energy of the system of particles in classical regime, is translationally invariant and does not distinguish between the "interior" and "exterior" of some "container". Thus $H(\Gamma)$ does not depend upon the volume $V$ of this container and so both quantities $P$ and $\Psi$ are formally identically equal to zero. But in fact any system in thermal equilibrium should be confined in space, so system's energy should depend upon the value of the volume $V$ - in the opposite case no pressure and dynamic compressibility may be formally defined at all.

\section{Solution of the problem}

In order to overcome this contradiction and to obtain the adequate definitions of $P$ and $\Psi$ it seems appropriate to act in the spirit of Bogolyubov's method [11] and, partly following Zubarev [12], to violate (perhaps, virtually) the translational symmetry of "free" Hamilton function $H(\Gamma)$. This is achieved rather simply by adding to $H(\Gamma)$ the singular repulsive potential $U_{\mathrm{V}}(q)$

$$
H_{\mathrm{V}}^{(\varepsilon)}(\Gamma)=H(\Gamma)+\varepsilon U_{\mathrm{V}}(q) ; \quad U_{\mathrm{V}}(q)=0 \quad\left(q \in S_{\mathrm{V}}\right), \quad U_{\mathrm{V}}(q) \rightarrow \infty \quad\left(q \in S_{\mathrm{V}}\right) .
$$

The potential $U_{\mathrm{V}}(q)$ is also referred to as the "contact delta-like", or "hard core" potential which dynamically describes the container of volume $V$ with the surface $S_{\mathrm{V}}$ confining the idealized "impenetrable" walls. Evidently, $U_{\mathrm{V}}(q)$ should be fully independent of the form of any actually present "wall-particle" interaction; the role of $U_{\mathrm{V}}(q)$ is to introduce - though in rather implicit form - the dependence of the $\varepsilon$ - deformed Hamilton function (5) on the volume $V$ as an external (!) fixed thermodynamic parameter.

By virtue of the suggested properties $(5)$ for $U_{\mathrm{V}}(q)$, the configuration part of $\Gamma$ is naturally divided into the "interior" and "exterior" parts (relative to the container) and $U_{\mathrm{V}}(q)$ itself acquires the properties of the generalized function; thus its correct handling ${ }^{4}$ (in particular, the differentiation) requires the use of some functionals (here - the partition function, see section 4).

Taking these definitions into account, it is natural to define the quantities $P$ and $\Psi$ in the proper and unambiguous way as the "quasidynamical" variables in the following "limiting" sense:

$$
\begin{gathered}
P_{\mathrm{V}}(\Gamma) \equiv \lim \left[-\partial H_{\mathrm{V}}^{(\varepsilon)}(\Gamma) / \partial V\right] \\
\Psi_{\mathrm{V}}(\Gamma) \equiv \lim \left[\partial^{2} H_{\mathrm{V}}^{(\varepsilon)}(\Gamma) / \partial V^{2}\right] \quad(\varepsilon \rightarrow 0) ; \quad \Psi_{\mathrm{V}}(\Gamma) \neq-\partial P_{\mathrm{V}}(\Gamma) / \partial V
\end{gathered}
$$

It should be stressed that the mathematical hallmark of the Bogolyubov method of quasiaverages [11] consists in their non-analytic dependence upon the infinitesimal parameter $\varepsilon$, and this is just the main reason why the results of the limiting procedure (6) drastically differ from the identically zero results for $P(\Gamma)$ and $\Psi(\Gamma)$, when $\varepsilon$ is taken equal to zero from the very beginning.

It can be shown (details of calculation see in [15], App. 7) that $P_{\mathrm{V}}(\Gamma)$ defined in (6) coincides exactly with the previously known result by Zubarev [12], whereas $\Psi_{\mathrm{V}}(\Gamma)$ in the form (6) was presented in [15] explicitly for the first time. It is worth noting that in [15] there was also obtained a quantum generalization of these results based on the well known Hellman - Feynman theorem for the operator's parameter differentiation.

Finally, averaging the $\Psi_{\mathrm{V}}(\Gamma)$ according to (1) gives $\Psi(\beta, V)$ and thus allows us to obtain in quite general way the solution of the long-standing and rather controversial problem [2-8] of thermal equilibrium pressure fluctuations (3) in isothermal-isochoric Gibbs ensemble for the nonideal systems of particles in classical regime.

In this paper, we will consider mainly the dynamical part of this problem $[15,16]$ which is intimately connected with the Bogolyubov method of quasi-observables of a purely dynamic origin. The thermodynamic part will also be presented (see section 7) but only for the simplest uniform case; more general cases may be found in [17-19] where some technical problems by calculation of the averages (1) of expressions (6) for a definite choice of $H(\Gamma)$ in $(5)$ are discussed.

${ }^{4}$ Possibly, just these circumstances have led to the failure of perturbation approaches in papers [2] and [6]. 


\section{Quasidynamical equations of state}

The key mathematical device in obtaining the quasidynamical equations of state (6) is the equality of volume derivatives of $n$-th order for two types of the well-defined functionals, namely $Z_{\mathrm{V}}(\beta)=\int \mathrm{d} \Gamma \exp [-\beta H(\Gamma)]$, where integration goes over the kinematically confined coordinate subspace of $\Gamma$ with volume $V$, and $Z_{\mathrm{V}}^{(\varepsilon)}(\beta)=\int \mathrm{d} \Gamma \exp \left[-\beta H_{\mathrm{V}}^{(\varepsilon)}(\Gamma)\right]$, where integration goes over the whole coordinate subspace of $\Gamma$ and only after this integration the limit $\varepsilon \rightarrow 0$ is taken.

For $n=0$ the equality is quite obvious because the dynamical factor $\Delta_{\mathrm{V}}(\Gamma)=\exp \left[-\beta U_{\mathrm{V}}(q)\right]$ acts as the projection operator onto the relevant coordinate subspace of $\Gamma$. Indeed, according to the definition (5) for the external wall potential $U_{\mathrm{V}}(q), \Delta_{\mathrm{V}}(\Gamma)=1$ if $U_{\mathrm{V}}(q)=0$ and $q$ belongs to the interior of the container, and $\Delta_{\mathrm{V}}(\Gamma)=0$ if $U_{\mathrm{V}}(q) \rightarrow \infty$ when $q$ belongs to the exterior of the container or even to its walls.

Details of calculations for $n=1$ and $n=2$ which provide a constructive realization of the definitions (6), can be found in the paper [15], App. 7; the main result is as follows. Suppose the macroscopic dynamic system is confined by the finite volume $V$ and is described by the Hamilton function of the form (5). Then the explicit expressions for $P_{\mathrm{V}}(\Gamma)$ and $\Psi_{\mathrm{V}}(\Gamma)$ are defined only by the "free" part $H(\Gamma)$ of the Hamilton function (5) and are quite independent ${ }^{5}$ of the specific form of the "wall potential" $U_{\mathrm{V}}(\Gamma)$.

Further, we perform the canonical scaling transformation in the phase space $\Gamma=\{q, p\} \rightarrow \Gamma_{\lambda}=$ $\{\lambda q, p / \lambda\}$, preserving the Liouville dynamic measure - i. e., the volume element of phase space, because $\mathrm{d} \Gamma_{\lambda}=(\lambda \mathrm{d} q)(\mathrm{d} p / \lambda)=(\mathrm{d} q \mathrm{~d} p)=\mathrm{d} \Gamma$. The auxiliary variable $\lambda$ establishes the connection between the change of the volume $V$ and the equivalent change of the coordinates $q$; clearly, the condition of canonicity also demands the corresponding change of the momenta $p$.

By these means we obtain the following expressions for $P_{\mathrm{V}}(\Gamma)$ and $\Psi_{\mathrm{V}}(\Gamma)$ in terms of the partial derivatives of the Hamilton function $H\left(\Gamma_{\lambda}\right)$ for the "free", or unconfined, system but with $\lambda$-deformed phase space variables:

$$
\begin{gathered}
P_{\mathrm{V}}(\Gamma)=-\left.(1 / f V)\left[D_{\lambda} H\left(\Gamma_{\lambda}\right)\right]\right|_{\lambda=1}, \\
\Psi_{\mathrm{V}}(\Gamma) \equiv(1 / V) P_{\mathrm{V}}(\Gamma)+\Delta \Psi_{\mathrm{V}}(\Gamma), \quad \Delta \Psi_{\mathrm{V}}(\Gamma)=\left.(1 / f V)^{2}\left[D_{\lambda}\left(\mathbf{1}+D_{\lambda}\right) H\left(\Gamma_{\lambda}\right)\right]\right|_{\lambda=1} .
\end{gathered}
$$

Here $D_{\lambda} \equiv \mathrm{d} / \mathrm{d} \lambda$, and $\mathbf{1} \equiv D_{\lambda}^{0}$ is the symbolic designation of the unity operator in the operator family $\left\{D_{\lambda}^{n}\right\}(n \geqslant 0$ - integer $) n$-fold differentiation on $\lambda$, after which one should put everywhere $\lambda=1$. Expressions (7) are well-defined for sufficiently smooth ${ }^{6}$ Hamilton function $H(q, p)-$ i. e., twice differentiable upon the arguments $p$ and $q$, while this operation does not anyhow effect the dependence of $P_{\mathrm{V}}(\Gamma)$ and $\Psi_{\mathrm{V}}(\Gamma)$ upon $V$.

Note that the terms of different order in $D_{\lambda}$ entering $\Delta \Psi_{\mathrm{V}}(\Gamma)$ may give contributions of the same order; e. g., in the case (8) (see below) for $H_{\mathrm{k}}(p)$ the terms in $(7)$ take the form ${ }^{7}$ :

$$
\begin{gathered}
{\left.\left[D_{\lambda} H_{\mathrm{k}}(p / \lambda)\right]\right|_{\lambda=1}=-m H_{\mathrm{k}}(p),\left.\quad\left[D_{\lambda}^{2} H_{\mathrm{k}}(p / \lambda)\right]\right|_{\lambda=1}=m(m+1) H_{\mathrm{k}}(p),} \\
P_{\mathrm{V}}(p)=(1 / f V) m H_{\mathrm{k}}(p), \quad \Delta \Psi_{\mathrm{V}}(p)=(1 / f V)^{2} m^{2} H_{\mathrm{k}}(p) .
\end{gathered}
$$

The expression for $P_{\mathrm{V}}(\Gamma)$ in (7) is usually cited as the Bogolyubov-Zubarev theorem $[11,12]$, whereas the expression for $\Psi_{\mathrm{V}}(\Gamma)$ was obtained in the paper by Rudoy and Sukhanov [15]. Expressions (7) are natural to be referred to as the (quasi)dynamic equations of state, because they connect the (quasi)dynamic quantities - pressure $P(\Gamma)$ and compressibility $\Psi(\Gamma)$ with the main energetic characteristic of the dynamical system - the Hamilton function $H(\Gamma)$.

It is essential that the dynamic equations of state (7) do not include an external thermal parameter - the temperature $T$, but they contain the explicit dependence upon the external mechanical parameter - the volume $V$; clearly, all the dynamic functions $H, P$ and $\Psi$ are defined on the system's phase space $\Gamma$. Note that all functions entering (7) are usually (but not always!) additive, so their average values are proportional to particle's number $N$.

\footnotetext{
${ }^{5}$ The situation here is quite similar to the well-known (see [14]) for the simplest generalized function - i. e., onedimensional Dirac $\delta$-function: the results of calculation do not depend on the concrete form of limit sequence for it.

${ }^{6}$ Note once more that the singular wall potential $U_{\mathrm{V}}(q)$ in no way enters the final expressions (7).

${ }^{7}$ Just the same expressions will be true for the contribution into $(7)$ from $H_{\mathrm{p}}(q)$ with the replacement of $m$ by $l$.
} 
Moreover, functions entering (7) possess various - but universal for all dynamical systems kinds of behavior relative to the volume $V$, namely $H(\Gamma)=O\left(V^{0}\right), P_{\mathrm{V}}(\Gamma)=O\left(V^{-1}\right), \Psi_{\mathrm{V}}(\Gamma)=$ $O\left(V^{-2}\right)$. Indeed, the external parameter $V$ enters the right-hand parts of $(7)$ only in the denominator, so in the limit $V \rightarrow \infty$ (i. e., for the case of "free" system) quantities $P_{\mathrm{V}}(\Gamma)$ and $\Psi_{\mathrm{V}}(\Gamma)$ really tend to zero in full accord with the previous arguments, whereas $H_{\mathrm{V}}(\Gamma)$ stays invariable in this limit.

For most non-ideal macroscopic systems Hamilton function $H(q, p)$ is of additive and separable nature in $q$ and $p$, so they may be presented as the sum of three terms: constant rest energy $E_{0}$, kinetic energy $H_{\mathrm{k}}(p)$ and potential energy $H_{\mathrm{p}}(q)$. These energies are usually additive relative to all the particles $\left(E_{0}\right.$ and $\left.H_{\mathrm{k}}(p)\right)$ and to their pairs $\left(H_{\mathrm{p}}(q)\right)$; evidently, the energy $E_{0}$ gives no contribution in the equations (7) to the pressure $P$ and compressibility $\Psi$ - as it should be.

\section{Uniform ideal and non-ideal case}

Uniform non-ideal case. In [15] the particular case was considered where both energies $H_{\mathrm{k}}(p)$ and $H_{\mathrm{p}}(q)$ are uniform (in Euler's sense) functions of their arguments with exponents $m$ and $l$ accordingly. This means that

$$
H_{\mathrm{p}}(\lambda q)=\lambda^{l} H_{\mathrm{p}}(q), \quad H_{\mathrm{k}}\left(\lambda^{-1} p\right)=\lambda^{-m} H_{\mathrm{k}}(p),
$$

so the expressions (7) acquire accordingly the forms

$$
\begin{aligned}
P_{\mathrm{V}}(q, p) & =(1 / f V)\left[m H_{\mathrm{k}}(p)-l H_{\mathrm{p}}(q)\right], \\
\Delta \Psi_{\mathrm{V}}(q, p) & =(1 / f V)^{2}\left[m^{2} H_{\mathrm{k}}(p)+l^{2} H_{\mathrm{p}}(q)\right] .
\end{aligned}
$$

It is worth noting that entering the right-hand side of the expression for $P_{\mathrm{V}}(q, p)$ in $(9)$ quantity $\left(-l H_{\mathrm{p}}(q)\right)=q F(q)$, where $F(q)=-\partial H_{\mathrm{p}}(q) / \partial q$, has the meaning of the Clausius force virial; then after the Gibbs averaging the resulting expression is nothing else but the virial theorem. Note, that in this approach it is not necessary to invoke the dynamical equations of motion with the additional assumptions of their stationarity relative to the time averaging: here we operate only with the phase space variables without the explicit use of time variable.

The "uniform" expressions (8) and (9) possess the following useful properties.

1. At any non-zero exponents $m$ and $l$ in (8) both energies $H_{\mathrm{k}}(p)$ and $H_{\mathrm{p}}(q)$ linearly enter the right-hand parts of (8), and every differentiation on $\lambda$ increases both $m$ and $l$ by unity.

2. Physical dimensionality for the pressure in (9) corresponds to the energy volume density whereas for the dynamic compressibility in (9) - the same for the pressure volume density, and every differentiation on $\lambda$ increases the power of the factor $1 / V$ by unity.

3. There exist conditions when the pressure $P_{\mathrm{V}}(q, p)$ as well as the compressibility $\Psi_{\mathrm{V}}(q, p)$ are proportional to the full energy $H(q, p)=H_{\mathrm{k}}(p)+H_{\mathrm{p}}(q)$. At these conditions, according to (9), the average value $\langle\Psi\rangle$ is proportional to $\langle P\rangle$ and/or $\langle H\rangle$; thus $\langle\Psi\rangle$ is the usual thermodynamic average and its calculation does not present any additional problem. Clearly, these conditions can be fulfilled only in two cases: at $m=-l$ or at $l=0$, while $m$ may be arbitrary.

In a series of papers [15-19] we were confined by the case of ideal dynamic system, where there is no coordinate-dependent potential energy $H_{\mathrm{p}}(q)$ of the interparticle interaction $(l=0)$. The full energy $H(q, p)$ for this system is presented by the sum of the constant term $E_{0}$ and of the kinetic energy $H_{\mathrm{k}}(p)$ which depends only on the particle's momenta:

$$
H_{\mathrm{p}}(q)=0, \quad H(\Gamma) \equiv H(q, p)=E_{0}+H_{\mathrm{k}}(p)
$$

Uniform ideal gas. In the case when $H_{\mathrm{k}}(p)$ is a uniform function (in Euler's sense) with the exponent $m$, expressions (8) acquire the following simple form:

$$
P_{\mathrm{V}}(p)=\mu\left[H_{\mathrm{k}}(p) / V\right], \quad \Delta \Psi_{\mathrm{V}}(p)=(1 / V) \mu P_{\mathrm{V}}(p)=(1 / V) \mu^{2}\left[H_{\mathrm{k}}(p) / V\right], \quad \mu \equiv m / f .
$$


Note that in both expressions (11) enters the constant $\mu=m / f$ which is equal to the ratio of the uniformity exponent $m$ to the number of the degrees of freedom $f$. The ratio $\mu$ characterizes the given dynamical system in the course of its dynamic (as well as thermodynamic) description in both classic and quantum regimes; thus, $\mu$ represents some kind of "similarity index" and thus specifies the whole class of dynamical systems.

For the given values of $f=1,2,3$ typical values of index $\mu$ may vary from $\mu_{\mathrm{nr}}=m_{\mathrm{nr}} / f=2 / f$ up to $\mu_{\mathrm{ur}}=m_{\mathrm{ur}} / f=1 / f$, where the subscripts "nr" and "ur" correspond to the non- and ultra-relativistic limits for the kinetic energy $H_{\mathrm{k}}(p)$ :

$$
H_{\mathrm{k}}^{\mathrm{nr}}(p) \approx(c p)^{2} / 2 E_{0} \quad\left(c p / E_{0} \ll 1\right), \quad H_{\mathrm{k}}^{\mathrm{ur}}(p) \approx c p \quad\left(c p / E_{0} \gg 1\right) ;
$$

in a particular case of massless particles (e. g., photons) with $E_{0}=0$ the expression for $H_{\mathrm{k}}^{\mathrm{ur}}(p)$ becomes exact. Obviously, in both limiting cases in (12) the kinetic energy is of the form

$$
H_{\mathrm{k}}(p)=\alpha_{m} p^{m}, \quad m_{\mathrm{ur}}=1, \quad \alpha_{1} \equiv \alpha_{\mathrm{ur}}=c ; \quad m_{\mathrm{nr}}=2, \quad \alpha_{2} \equiv \alpha_{\mathrm{nr}}=\left(\alpha_{1}\right)^{2} / 2 E_{0},
$$

which is the exponential - and thus uniform (in Euler's sense) - function of the momentum $p$ with the uniformity exponent $m$ equal to 2 and 1 correspondingly.

More general, for any possible values $1 \leqslant f \leqslant 3$ and $1 \leqslant m \leqslant 2$ one gets $1 / 3 \leqslant \mu \leqslant 2$, but in some models of the "ideal gas" (e. g., used in modern cosmology) the intervals of the change of the parameters $m, f$ and $\mu=m / f$ may differ in magnitude (and sometimes also in sign); nevertheless, the expressions (11) preserve the applicability for these cases too.

It is noticeable, that if the energy density is positive the pressure fluctuations are also positive (being independent of the sign of $\mu$ ); this means that the system may be mechanically stable $(\Delta \Psi>0)$ even if the pressure is negative $(P<0)$. Moreover, this is just the case if $\mu<0$ due to an unusual value $m<0$ (e. g., for the Chaplygin gas); note that the condition $f>0$ is always fulfilled by definition.

\section{Non- and quasi-uniform ideal gas}

Non-uniform ideal gas. More general case of the ideal gas was considered in [16,17], namely the case of the free isotropic relativistic particles with non-uniform Hamilton function $H(p)$ consisting of the rest energy $E_{0} \equiv H(0)$ and of the kinetic energy $H_{\mathrm{k}}(p)$ with $H_{\mathrm{k}}(0)=0$.

The expression for $H(p)$ is usually given by the Lorentz-Einstein equation

$$
H(p) \equiv E_{0}+H_{\mathrm{k}}(p)=\left[E_{0}^{2}+(c p)^{2}\right]^{1 / 2}, \quad H(p)=E_{0} h(p), \quad h(p)=1+h_{\mathrm{k}}(p) ;
$$

which is convenient to rewrite in dimensionless form:

$$
h(\xi)=1+h_{\mathrm{k}}(\xi)=\left(1+\xi^{2}\right)^{1 / 2} ; \quad h_{\mathrm{k}}(\xi)=h(\xi) f \mu^{(-)}(\xi), \quad \xi=c p / E_{0} \quad\left(E_{0} \neq 0\right) ;
$$

here $c$ is the velocity of light in vacuum, $h$ and $h_{\mathrm{k}}-$ the dimensionless energies ${ }^{8}$ (full and kinetic).

The dynamic equations of state follow immediately from (7) but differ noticeably from (11). Using the dimensionless variable $\xi=c p / E_{0}$, we get instead of (11) the following exact dynamical equations of state

$$
\begin{aligned}
P_{\mathrm{V}}(\xi) & =\left(E_{0} / f V\right)\left\{\left[h^{2}(\xi)-1\right] / h(\xi)\right\}=[H(\xi) / V] \nu^{(-)}(\xi)=\left[H_{\mathrm{k}}(\xi) / V\right] \mu^{(+)}(\xi), \\
\Delta \Psi_{\mathrm{V}}(p) & =E_{0}(1 / f V)^{2}\left\{\left[h^{4}(\xi)-1\right] / h^{3}(\xi)\right\}=(1 / V) P_{\mathrm{V}}(\xi) \nu^{(+)}(\xi) \\
& =(1 / V)\left[H_{\mathrm{k}}(\xi) / V\right] \mu^{(+)}(\xi) \nu^{(+)}(\xi) .
\end{aligned}
$$

It is worth noting that the non-uniform expressions (14)-(16) are much more complicated compared to their uniform counterparts (11). In particular, instead of the unique and constant "similarity

\footnotetext{
${ }^{8}$ In the case $E_{0}=0$ the ultra-relativistic limit becomes an exact one and becomes a uniform case with $m=1$ (see equation (13)).
} 
index" $\mu$ in (11) one obtains in (14)-(16) the whole family of the variable dimensionless factors $\nu^{( \pm)}(\xi)$ and $\mu^{( \pm)}(\xi)$. These factors have the meaning of the generalized "similarity indices" and depend (though weakly enough) on $\xi$ by means of the function $h(\xi)$ :

$$
\begin{gathered}
f \mu^{( \pm)}(\xi)=1 \pm[h(\xi)]^{-1}, \quad f \nu^{( \pm)}(\xi)=1 \pm[h(\xi)]^{-2}, \quad f \kappa^{( \pm)}(\xi)=1 \pm[h(\xi)]^{-4} \\
f \mu^{(+)}(\xi) \mu^{(-)}(\xi)=\nu^{(-)}(\xi), \quad f \nu^{(-)}(\xi) \nu^{(+)}(\xi)=f \kappa^{(-)}(\xi) \\
h_{\mathrm{k}}(\xi)=h(\xi) f \mu^{(-)}(\xi) .
\end{gathered}
$$

The system of exact equations (14)-(17) is rather complicated, but in practice only their approximate forms are of real interest - i. e., two limiting cases of them: the non-relativistic $\left({ }^{\mathrm{nr}}\right)$ $(\xi \rightarrow 0)$ and the ultra-relativistic $\left({ }^{\mathrm{ur}}\right)(\xi \rightarrow \infty)$ ones. The lowest order corrections to the functions $h_{\mathrm{k}}(\xi)$ and $1 / h_{\mathrm{k}}(\xi)$, compared to their "uniform" analogs (12) and (13), are of the form:

$$
\begin{gathered}
h_{\mathrm{k}}(\xi) \approx h_{\mathrm{k}}^{\mathrm{nr}}(\xi)\left[1-(1 / 4) \xi^{2}\right]=h_{\mathrm{k}}^{\mathrm{nr}}(\xi)\left[1-(1 / 2) h^{\mathrm{nr}}(\xi)\right], \quad h_{\mathrm{k}}^{\mathrm{nr}}(\xi)=(1 / 2) \xi^{2} \quad(\xi \rightarrow 0), \\
1 / h_{\mathrm{k}}(\xi)=\left[h_{\mathrm{k}}^{\mathrm{ur}}(\xi)\right]^{-1}\left\{1-(1 / 2) \xi^{2}\right\}=\left[h_{\mathrm{k}}^{\mathrm{ur}}(\xi)\right]^{-1}\left\{1-(1 / 2)\left[h_{\mathrm{k}}^{\mathrm{ur}}(\xi)\right]^{-2}\right\}, \\
{\left[h_{\mathrm{k}}^{\mathrm{ur}}(\xi)\right]^{-1}=\xi^{-1} \quad(\xi \rightarrow \infty) .}
\end{gathered}
$$

Note that $h_{\mathrm{k}}^{\mathrm{nr}}(0)=1 / h_{\mathrm{k}}^{\mathrm{ur}}(\infty)=0$, which allows us to consider the quantities $h_{\mathrm{k}}(\xi)$ and $1 / h_{\mathrm{k}}(\xi)$ as small in corresponding regions of the change of the variable $\xi$.

In some physical problems, there may be of interest to obtain the corrections to the limiting "uniform" equations (11) and (12), which are stipulated by the variable nature of functions $\kappa^{( \pm)}(\xi)$ and $\mu^{( \pm)}(\xi)$, entering the dynamical equations of state $(16)$ and $(17)$ for $P(\xi ; V)$ and $\Psi(\xi ; V)$. In order to carry out the procedure in the spirit of perturbation theory at small values $\xi$ " 1 in nonrelativistic $\left({ }^{\mathrm{nr}}\right)$ limit and at large values $\xi^{\prime \prime} 1$ in ultra-relativistic ( ${ }^{\mathrm{ur}}$ ) limit it is convenient to use in equations (16)-(18) as small parameters not $\xi$ and $1 / \xi$, but the quantities $h_{\mathrm{k}}(\xi)$ and $1 / h_{\mathrm{k}}(\xi)$.

Omitting the simple but lengthy calculations, one obtains the following approximate results:

$$
\begin{gathered}
P_{\mathrm{V}}(\xi) \approx \mu_{\mathrm{nr}}\left[H_{\mathrm{k}}(\xi) / V\right]\left[1-(1 / 2) h_{\mathrm{k}}(\xi)\right], \quad \Delta \Psi_{\mathrm{V}}(\xi) \approx \mu_{\mathrm{nr}}\left[H_{\mathrm{k}}(\xi) / V\right]\left[1-(3 / 2) h_{\mathrm{k}}(\xi)\right](\xi \rightarrow 0) \\
P_{\mathrm{V}}(\xi) \approx \mu_{\mathrm{ur}}\left[H_{\mathrm{k}}(\xi) / V\right]\left\{1+\left[h_{\mathrm{k}}(\xi)\right]^{-1}\right\}, \quad \Delta \Psi_{\mathrm{V}}(\xi) \approx \mu_{\mathrm{ur}}\left[H_{\mathrm{k}}(\xi) / V\right](\xi \rightarrow \infty)
\end{gathered}
$$

these expressions reveal the tendency to the "sloping" of the dependence upon $\xi$ both kinematic $\left(\kappa^{(+)}, \mu^{(+)}\right)$and dynamic $\left(h_{\mathrm{k}}, P, \Delta \Psi\right)$ quantities: at small (but finite) $\xi$ all these quantities become smaller comparing their "uniform" limits at $\xi=0$, whereas at large (but finite) $\xi$ - on the contrary, larger comparing their "uniform" limit at $1 / \xi=0$.

Quasi-uniform ideal gas. The most general case of ideal gas includes the Hamilton function $H(p)$ with the non-uniform dependence upon $p$. But in practice only certain limiting cases (e. g., non- or ultra-relativistic ones) are of interest, where $H(p)$ (and hence its derivatives) may be presented as an expansion in integer powers $m$ of $p$ with $m>0$ or $m<0$ (i. e., in $1 / p$ ), where $H_{0}(p)=h_{0} \equiv E_{0}=$ const, $m_{0} \equiv 0$, but $m_{i}$ and $h_{i}$ at $i=1,2, \ldots$ may be of both signs:

$$
\begin{aligned}
H(p) & =\sum_{i=0}^{n} H_{i}(p)=\sum_{i=0}^{n} H_{i}(p)=\sum_{i=0}^{n} h_{i} p^{m_{i}} \\
P_{\mathrm{V}}(p) & =(1 / f V) \sum_{i=0}^{n} m_{i} H_{i}(p)=(1 / f V) \sum_{i=0}^{n} m_{i} h_{i} p^{m_{i}} \\
\Psi_{\mathrm{V}}(p) & =(1 / f V)^{2} \sum_{i=0}^{n} m_{i}^{2} H_{i}(p)=(1 / f V)^{2} \sum_{i=0}^{n} m_{i}^{2} h_{i} p^{m_{i}}
\end{aligned}
$$

Obviously, every term in (22) is a uniform one, whereas the whole expression (22) is not; so it may be considered as a quasi-uniform one and characterized not by the single uniformity exponent 
but by the whole discrete set of them - the examples may be found in (18) and (19):

$$
\begin{array}{lll}
\text { NR-limit: } m_{1}=2, \quad h_{1}>0 ; & m_{2}=4, \quad h_{2}=-(1 / 4) h_{1}<0 ; \\
\text { UR-limit: } m_{1}=1, \quad h_{1}>0 ; \quad m_{2}=-1, \quad h_{2}=(1 / 2) h_{1}>0 .
\end{array}
$$

The final sign of the quantities presented in (22) is determined by the non-trivial interplay of the coefficients $h_{i}$ and $m_{i}$; as a rule, $h_{i}$ contain some small parameter and decrease in magnitude with increasing $i$, whereas $m_{i}$, on the contrary, increases with $i$ in magnitude.

It is worth noting that nowadays the Lorentz-Einstein expression (13) is not the uniquely possible one and therefore in [18] all the scheme outlined in this paper was carried out for this more general case. In particular, it appears, that in the Lorentz-violated case the UR-limit in (23) is supplemented by the third term with $h_{3}>0, m_{3}=2$, being typically of NR-form.

This term enters the equations (22) due to the appearance in the Lorentz-violated case of a new parameter $H(p) / E_{\mathrm{Pl}}\left(E_{\mathrm{Pl}}\right.$ is the universal Planck energy) which is always small - even in the extreme UR-situation when $H(p) / E_{0}$ is large; in other words, the ratio $E_{0} / E_{\mathrm{Pl}}$ is always very small for any reasonable choice of particles constituting the dynamical system. The analysis of relevant expressions shows the existence of some critical value $p^{*}$ defined as $c p^{*} \sim\left(E_{0}^{2} E_{\mathrm{Pl}}\right)^{1 / 3}$; when the particle's momentum achieves $p^{*}$ the usual Lorentz behavior ceases and velocity $v(p)=\mathrm{d} H(p) / \mathrm{d} p$ exceeds the critical value $c$ (details may be found in [18]).

\section{Thermodynamic equations of state}

As it was meant in section 2, the calculation of equilibrium pressure fluctuations (3) in terms of $\beta$ and $V$ (and, perhaps, $N$ ) will be completed after the averaging of the quasi-dynamic quantities obtained in sections $4-6$. This task is much more traditional but far from being simple, so we give here only the general outline for the ideal system for the case (14) of the non-uniform kinetic Hamilton function $H_{\mathrm{k}}(p)$. The partition function is of the multiplicative form

$$
\begin{aligned}
& Z_{N}(\beta, V)=[V z(\beta)]^{N}, \quad z(\beta)=\exp \left(-\beta E_{0}\right) z_{\mathrm{k}}(\beta), \\
& z_{\mathrm{k}}(\beta)=\int \mathrm{d} \Gamma(p) \exp \left[-\beta H_{\mathrm{k}}(p)\right] ; \\
& \rho_{\mathrm{k}}(p)=\left[z_{\mathrm{k}}(\beta)\right]^{-1} \exp \left[-\beta H_{\mathrm{k}}(p)\right], \quad \mathrm{d} \Gamma(p)=A_{f} p^{f-1} \mathrm{~d} p, \\
& \left(A_{1}=1, \quad A_{2}=2 \pi, \quad A_{3}=4 \pi\right) .
\end{aligned}
$$

This problem in somewhat less general formulation was partially considered in [15-19] on the grounds of original studies [20], but the results may not be always presented in the analytical form. The following terminology seems appropriate in order to analyze the limiting cases for the canonical distribution functions $\rho_{\mathrm{k}}(p)$ in $(24)$. For the general relativistic case (13) it is the Jüttner distribution (1911), for the non- and ultra-relativistic cases (12) - Maxwell distribution (1859) and Wien distribution (1896) accordingly.

The limiting cases (12) correspond to the normal (Gaussian, $m=2)$ and exponential $(m=1)$ distributions on $f$-dimensional random variable $p$; the general power case may be connected with gamma-distribution, but was seemingly not yet considered in physical applications.

The Massieu-Planck potential corresponding to ideal system (24) is of the form

$$
\Phi_{N}(\beta, V)=\ln Z_{N}(\beta, V)=N\left[\ln V-\beta E_{0}+\ln z_{\mathrm{k}}(\beta)\right],
$$

so the thermic equations of state (2) for any - not only uniform - dependence $H_{\mathrm{k}}(p)$ get the well known Clapeyron-Mendeleev equation of state:

$$
\begin{aligned}
P(\beta, V) & =N / \beta V=n k_{\mathrm{B}} T, \quad(n=N / V), \\
\chi(\beta, V) & =-(1 / V) P(\beta, V) ;
\end{aligned}
$$

unfortunately, the quantity $\Psi(\beta, V)$ (as mentioned above) cannot be obtained from (25) and needs the direct independent calculation. 
Uniform ideal case. Only in this particular case there is no such a need due to the simple and convenient relation (11) between $\Delta \Psi_{\mathrm{V}}(p)$ and $P_{\mathrm{V}}(p)$. Averaging (11) following the Gibbs prescription (1) with the canonical distribution function $\rho_{\mathrm{k}}(p)$ from $(24)$ and using the definition $(7)$ for $\Psi_{\mathrm{V}}(p)$, we arrive at the relations

$$
\begin{aligned}
\Psi(\beta, V) & =(1 / V) P(\beta, V)+\Delta \Psi(\beta, V), \\
\Delta \Psi(\beta, V) & \equiv\left\langle\Delta \Psi_{\mathrm{V}}(p)\right\rangle=(1 / V) \mu P(\beta, V) .
\end{aligned}
$$

Then we substitute the equations (26) and (27) into the basic equation (3) and thus obtain the desired expression for the equilibrium pressure fluctuations (more precisely, the absolute dispersion):

$$
\left\langle(\Delta P)^{2}\right\rangle=(1 / \beta)[\chi(\beta, V)+\Psi(\beta, V)]=(1 / \beta) \Delta \Psi(\beta, V)=(1 / \beta V) \mu P(\beta, V) ;
$$

note that the terms $(1 / V) P(\beta, V)$ stem in $(28)$ from $\chi(\beta, V)$ and $\Psi(\beta, V)$ with opposite signs and therefore exactly compensate one another. From (28) and (26) one can easily obtain the expression for the relative pressure fluctuations which shows the standard $1 / \sqrt{ } N$ behavior:

$$
\left\{\left\langle(\Delta P)^{2}\right\rangle / P^{2}(\beta, V)\right\}^{1 / 2}=\mu^{1 / 2} N^{-1 / 2} .
$$

Finally, it is of interest to obtain very simply not only the thermic equations of state (26) but also the caloric equations of state for the average energy $H(\beta)$ and the heat capacity $C_{\mathrm{V}}(\beta)$,

$$
\begin{aligned}
H(\beta) & =E_{0}+\left\langle H_{\mathrm{k}}(p)\right\rangle=E_{0}-N(\partial / \partial \beta) \ln z_{\mathrm{k}}(\beta), \\
C_{\mathrm{V}}(\beta) & =N\left(k_{\mathrm{B}} \beta^{2}\right)^{-1}\left(\partial^{2} / \partial \beta^{2}\right) \ln z_{\mathrm{k}}(\beta) .
\end{aligned}
$$

In order to avoid the more or less difficult differentiation on $\beta$, one may use the linear relation (11) between the pressure and energy; after averaging the expressions (10) and (11) one has:

$$
\begin{aligned}
\langle P(p)\rangle & =\mu\left\langle H_{\mathrm{k}}(p)\right\rangle / V, \quad H(\beta)-E_{0}=V(1 / \mu) P(\beta, V), \\
H(\beta) / N & =E_{0}+1 / \mu \beta, \quad C_{\mathrm{V}}(\beta) / N k_{\mathrm{B}}=1 / \mu=\text { const. }
\end{aligned}
$$

Here the first of equation $(26)$ for $\langle P(p)\rangle \equiv P(\beta, V)$ with the definition $\left\langle H_{\mathrm{k}}(p)\right\rangle \equiv H(\beta)-E_{0}$ were used; the last two relations in (31) may be considered as the slightly generalized form of the equipartition theorem for the arbitrary value of $\mu=m / f$ in case of the uniform function $H_{\mathrm{k}}(p)$.

Of course, just the same results for $H(\beta)$ and $C_{\mathrm{V}}(\beta)$ may be also obtained directly from $(30)$, but only if one previously calculates $z_{\mathrm{k}}(\beta)-$ e. g., by means of the Mellin integral transform:

$$
z_{\mathrm{k}}(\beta)=A_{f} \int_{0}^{\infty} \mathrm{d} p p^{f-1} \exp \left[-\beta \alpha_{m} p^{m}\right]=A_{f}(1 / m) \Gamma[1 / \mu]\left(\beta \alpha_{m}\right)^{-1 / \mu}
$$

Here $\Gamma[1 / \mu]$ is the gamma-function of the positive argument $1 / \mu$ and numerical factors $A_{f}$ are defined in (24); most interesting cases of three-dimensional $(f=3)$ non- $(m=2)$ and ultra$(m=1)$ relativistic limits in (32) correspond to the values $\mu=2 / 3$ and $\mu=1 / 3$.

The consecutive thermodynamic calculation according to expressions (24)-(26) for more difficult - i. e., non- and quasi-uniform - cases can be found in the papers $[18,19]$. In these cases the exact calculation is seemingly not possible and only the perturbative results in low- or high-temperature limits are accessible which correspond to non- or ultra-relativistic expansion in (18)-(21).

\section{Conclusion}

It was the aim of this paper to fully revise the problem of calculation of the equilibrium pressure fluctuations $\left\langle(\Delta P)^{2}\right\rangle$ in the framework of the Gibbs statistical mechanics. In section 1 the early attempts to solve the problem and the reasons for their failure are discussed, and in section 2 the precise formulation of the problem is given. In particular, it is shown that the difficulties with the 
pressure fluctuation - as compared, e. g., with the energy $H$ or generalized force $A$ fluctuations are of two main origins.

Firstly, both the relevant conjugate variables - i. e., pressure $P$ and volume $V$, are of dual nature - namely, dynamic as well as thermodynamic ones. Secondly, $V$ enters the exponent of the Gibbs canonical distribution function in the highly non-trivial (i. e., implicit and singular) way in contrast to the conjugate pairs $H$ and $\beta$ as well as $A$ and $a$; in the last two cases the conjugate variables are mutually independent and enter the bilinear products $-(\beta H)$ and $-(A a)$.

This fact explains why by calculation of $\left\langle(\Delta P)^{2}\right\rangle$ there should be taken account of both terms in the Gibbs lemma - i. e., the usual relevant thermodynamic susceptibility (in this case it is the always negative isothermic compressibility) as well as the average value of $\Psi$ - the variable which Gibbs called the dynamic compressibility.

In section 3 it is shown that the only correct and unambiguous way of finding $\Psi$ is to use the concept of quasiaverage (here - quasidynamic) quantities introduced by Bogolyubov in 1961. All these "quasi"-quantities are intended to overcome the well known general feature of the Gibbs statistical mechanics - namely, the possible occurrence of some kind of "degeneration" (classical as well as quantum) when the symmetry of the thermal equilibrium state is lower than the symmetry of the Hamilton function (as well as the Hamiltonian) $H$.

In section 4 the quasidynamic equations of state are found in the form of explicit expressions connecting $P$ and $\Psi$ with the first and second derivatives of $H$ and in sections 5 and 6 various examples are considered. In particular, the important role of the property of uniformity of $H$ (in the Euler's sense) is shown and various cases are studied in detail: uniform as well as non- and quasi-uniform; both cases are limited by ideal systems with account of only kinetic energy which may be of Lorentz as well as of non-Lorentz form.

Finally, in section 7 the thermodynamic equations of state for averages of $P$ and $\Psi$ are discussed and explicit calculation for $\left\langle(\Delta P)^{2}\right\rangle$ is consecutively carried out for the particular case of an ideal uniform system and the references to more complicated cases are given.

Let us conclude this article with the discussion of some issues of practical significance. First of all, this is the physical setting of the whole problem of the pressure and its fluctuations in the thermodynamic context of the closed system with $N=$ const. Here we have chosen the most convenient and commonly used case - i. e., the isochoric-isothermic one ( $V=$ const, $T=$ const), when the conjugate variables - pressure $P$ and energy $E$ may fluctuate.

Of course, it is of interest how these results will change for other possible choices of external conditions, and thus for Gibbs ensembles different from the canonical one - this problem is discussed in a very informative paper [21] as well as in our paper [15]. Unfortunately, we have missed the paper [21], so the present author is indebted to the referee for pointing this paper to him along with some earlier publications on the theme cited therein, especially the book by Rowlinson [22].

In particular, for the original Fowler's problem - i. e., for the case of ideal gas in canonical ensemble - it follows from equation (11) that $\left(\Delta P^{2}\right)$ is proportional to $\left(\Delta E^{2}\right)$ (where $E$ is in this case only kinetic energy), which coincides with the equation (4.2) in [21]. Of course, as is stated in [21], in the microcanonical ensemble due to the very definition both quantities $\left(\Delta E^{2}\right)$ and $\left(\Delta P^{2}\right)$ become equal to zero.

In our paper [15] we have also discussed the isobaric-isothermic situation $(P=$ const, $T=$ const), where $\left(\Delta P^{2}\right)$ is equal to zero by definition, as well as the isoentropic situation, when $S=$ const, whereas all the pairs of variables $P, V$ and $E, T$ may fluctuate. This situation is, perhaps, the most interesting one from the experimental point of view, but, unfortunately, it cannot be described in the framework of the Gibbs approach, because there is no relevant Gibbs ensemble for it.

Instead, one should use the Einstein approach of quasithermodynamic fluctuations (see, e. g., [10]), which for relative pressure fluctuations gives the following expression $\left(\Delta P^{2}\right) / \Delta P^{2}=$ $\left(\Delta E^{2}\right) / \Delta E^{2}+\left(\Delta V^{2}\right) / \Delta V^{2}$, which obviously exceeds the canonical Gibbs value $\left(\Delta P^{2}\right) / \Delta P^{2}=$ $\left(\Delta E^{2}\right) / \Delta E^{2}$ and is equal to the (not isothermic) adiabatic compressibility. We do not further discuss these issues here, because our primary goal was to show the consistency of the Gibbs approach with the problem of the pressure fluctuations by the aid of Bogolyubov's quasiaverages method. 


\title{
Acknowledgement
}

The author dedicate this paper to the honour of one of the greatest scientists of the twentieth century Nikolai Nikolaevich Bogolyubov on the occasion of his $100^{\text {th }}$ birthday. The author is indebted to Prof. Yu. P. Rybakov for valuable discussions and to Dr. I. Keita for assistance.

\section{References}

1. Gibbs J.W. Elementary Principles in Statistical Mechanics. Scribner, New Haven, 1902.

2. Fowler R.H. Statistical Mechanics. 2nd ed. Cambridge University Press, 1936.

3. Wergeland H. Det. Kgl. Norske Vidensk. Forh., 1955, 28, 106.

4. Münster A. - In: Termodinamika dei Processi Irreversibili. Scuola "Enrico Fermi” - X, Bologna, 1960; Nuovo Cimento Suppl., 1960, 13.

5. Münster A., Physica, 1960, 26, 1117.

6. Klein M. J., Physica, 1960, 26, 1073.

7. Hill T.L. Statistical Mechanics. McGraw-Hill, NY, 1956.

8. Kittel C. Thermal Physics. Wiley, New York, 1969.

9. Terletzky Ya.P. Statistical Physics, 2d ed. Vys. Shkola, Moscow, 1973 (in Russian).

10. Landau L.D., Lifshitz E.M. Statistical Physics. Addison-Wesley, Reading, Mass., 1958.

11. Bogolyubov N.N. Problems of Dynamical Theory in Statistical Physics. Gostekhizdat, Moscow - Leningrad, 1946, (in Russian).

12. Zubarev D.N. Statistische Thermodynamic der Nichtgleigewicht. Akademie Verlag, Berlin, 1976, § 5.3.

13. Bogolyubov N.N. Preprint R-1451 LTP, JINR, Dubna, 1961; Phys. Abh. aus der SU, 1962, 6, 1.

14. Vladimirov V.S. Generalized Functions in Mathematical Physics. Nauka, Moscow, 1976 (in Russian).

15. Rudoy Yu.G., Sukhanov A.D. Physics - Uspekhi, 2000, 43 (12), 1169.

16. Rudoy Yu.G., Keita I. Bulletin of People's Friendship University of Russia. Ser. Math., Inf. Sciences, Physics, 2007, 1-2, 84 (in Russian).

17. Rudoy Yu.G., Rybakov Yu.P., Keita I. Physical Education in Higher School, 2007, 13 (3), 41 (in Russian).

18. Rudoy Yu.G., Keita I. - In: Proc. of the International Conf. on Gravitation and Cosmology, RUSGRAV13, PFUR, Moscow, 2007 (in Russian).

19. Rudoy Yu.G., Rybakov Yu.P., Keita I. J. Math. Sci., 2009 (in press).

20. Jüttner F. Ann. Phys., 1911, 34, 856; ibid., 1928, 47, 542; Glaser W., Ann. Phys., 1935, 94, 317.

21. Ray J.R., Am. J. Phys., 1982, 50 (11), 1035.

22. Rowlinson J.S. Liquid and Liquid mixtures. Butterworth, Washington, DC, 1959, Ch. 8.

\section{Метод квазісередніх Боголюбова розв'язує задачу про Флуктуації тиску в статистичній механіці Гіббса}

\author{
Ю.Г. Рудой \\ Університет дружби народів, 117198, вул. Миклухо-Маклая, 6, Москва, Росія \\ Отримано 23 липня 2009 р., в остаточному вигляді - 3 вересня 2009 р.
}

\begin{abstract}
Цілком ревізовано давню та доволі нетривіальну задачу про розрахунок флуктуацій тиску в рівноважній статистичній механіці Гіббса. Критично проаналізовано попередні підходи та показано, що застосування ідей Боголюбова дає повний та недвозначний розв'язок цієї задачі. Ключову роль відіграє тут ідея Боголюбова про квазісередні (чи швидше квазідинамічні) величини - зокрема, тиск $P$ та динамічну стисливість $\Psi$. Віртуальне спряжене поле, яке порушує трансляційну інваріантність функції Гамільтона $H$ в границі $\varepsilon \rightarrow 0$, задається сингулярним потенціалом непроникних стінок контейнера. Отримано загальні співвідношення для $P$ та $\Psi$ у термінах похідних від $H$ та досліджено деякі приклади, в т.ч. випадки “ідеальної проти неідеальної” та "однорідної супроти не- або квазіоднорідної” (в Ейлеровому розумінні) функцій Гамільтона $H$, що описують систему.
\end{abstract}

Ключові слова: рівноважна статистична механіка Гіббса, квазісередні Боголюбова, флуктуації тиску, релятивістичний ідеальний газ

PACS: 05.70.-a, 05.30.-d, 05.40.-a 